\title{
Monika Paulat, Präsidentin des Landessozialgerichts Berlin-Brandenburg
}

\author{
Juristinnen machen Karriere - wir stellen sie vor
}

\begin{abstract}
Das Gespräch führte Ramona Pisal, Vizepräsidentin im Bundesvorstand und Vorsitzende des Landesverbands Brandenburg im djb, Vorsitzende Richterin am Oberlandesgericht, Brandenburg an der Havel, im April 2011 in Potsdam.
\end{abstract}

\begin{abstract}
Liebe Frau Paulat, seit dem 1. Juni 2009 sind Sie Präsidentin des Landessozialgerichts Berlin-Brandenburg mit Sitz in Potsdam. War das Ihr lang angestrebtes Ziel im Berufsleben und auf der Landkarte?

Der Weg nach Potsdam war lang und im Detail nicht geplant. Manchmal bin ich noch immer überrascht, dass ich hier gelandet bin. Auf jeden Fall freue ich mich, dass es so gekommen ist.
\end{abstract}

\section{Aber Jura studieren und Richterin werden - das wollten Sie schon immer?}

Juristin zu werden, war mir nicht in die Wege gelegt. In meiner Familie gab und gibt es weit und breit keine Juristen.

Mein Vater war Grundschul- und Sonderschullehrer, meine Mutter hatte eine Banklehre absolviert.

Eigentlich wollte ich meinem Vater nacheifern und Lehrerin werden, später auch Ärztin. Weil ich auf einen Studienplatz für Medizin hätte warten müssen und sehr gut in Deutsch war, entschloss ich mich nach dem Abitur, Jura in Frankfurt am Main zu studieren, wohin unsere Familie Anfang der 60er Jahre von Ostfriesland gezogen war.

Mein Vater unterstützte mich in der Studienwahl sehr, wie er überhaupt mein erster Förderer war. Es war eine gute Wahl. Schon im ersten Semester hatte ich im Einführungsseminar für Zivilrecht ein ermutigendes Erfolgserlebnis. Mir machte es große Freude, Sachverhalte unter Normen zu subsumieren, zu argumentieren, Meinungen und Gegenmeinungen abzuwägen und ein überzeugendes Ergebnis zu finden.

Während des gesamten Studiums war das Zivilrecht meine Lieblingsdisziplin.

Nach dem ersten Staatsexamen wollte ich einen Teil der Referendarzeit in meiner Heimat Ostfriesland verbringen. Zur Ausbildung kam ich in den Landgerichtsbezirk Aurich. Hier traf ich in der ersten Veranstaltung der Referendararbeitsgemeinschaft meine große Liebe und späteren Ehemann. Der Plan, wieder nach Hessen zurückzugehen, war bald begraben. Ich blieb in Ostfriesland, legte in Hannover die Große Juristische Staatsprüfung ab, wartete danach erst einmal auf die Einstellung in den Richterdienst.

Während des Referendariats hatte sich mein Wunsch, Richterin zu werden, entwickelt, Zivilrichterin wollte ich werden. Ich hatte beim Landgericht Aurich eine sehr prägende Station bei einem Zivilrichter absolviert.

\section{Wie sind Sie dann zur Sozialgerichtsbarkeit gekommen?}

Seinerzeit gab es in Niedersachsen einen Einstellungsstopp. Ich wartete eine ganze Weile, bis es plötzlich hieß, in der So-
Monika Paulat, geboren 1948 in Norden/Ostfriesland, studierte in Frankfurt am Main Rechtswissenschaft und legte 1976 in Hannover ihr zweites juristisches Staatsexamen ab. Sie begann ihren Richterdienst 1978 in Niedersachsen mit einer Einweisung am LSG Celle und nachfolgender Tätigkeit an den Sozialgerichten Oldenburg und Aurich. Nach früher Erprobung am LSG Celle war sie mit nur 36 Jahren eine junge Richterin am LSG. Dort wechselte sie bald als Präsidialrichterin in die Gerichtsverwaltung und sammelte Erfahrung als Justitiar- und Personaldezernentin sowie in der Leitung des Gerichts, bis sie mit der Ernennung zur Direktorin des Sozialgerichts in Oldenburg im Januar 1989 und anschließend ab 1992 des Sozialgerichts in Hannover selbst die Leitung eines Gerichts übernahm. 1996 wurde sie zur Präsidentin des Landessozialgerichts Bremen ernannt, anschließend (1999-2002) übte sie dieselbe Tätigkeit in Niedersachsen aus. Von 2002 bis 2009 leitete sie das Landessozialgericht Niedersachsen-Bremen, dessen Fusion sie maßgeblich begleitet hatte. Am 29. Mai 2009 wurde sie von Justizsenatorin Gisela von der Aue und der damaligen brandenburgischen Justizministerin Beate Blechinger zur Präsidentin des Landessozialgerichts Berlin-Brandenburg ernannt.

Monika Paulat ist verheiratet. Sie lebt mit ihrem Mann, der Notar ist, derzeit nur an einigen Wochenenden in Bremen, im Übrigen nahe ihrer Arbeitsstätte in Potsdam.

zialgerichtsbarkeit würden Richter eingestellt werden, Richterinnen natürlich auch.

Von der Sozialgerichtsbarkeit hatte ich noch nie etwas gehört. Bekannt war mir lediglich die Existenz der Reichsversicherungsordnung, immerhin, und eine bestimmte Vorschrift,

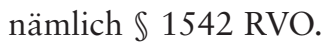

Ich stellte mich in Celle beim Landessozialgericht und im Justizministerium vor. Der Präsident des Landessozialgerichts war froh, jemanden einstellen zu können, der an das Sozialgericht Aurich gehen würde, denn dafür gab es nicht besonders viele Interessenten.

Ich zog also im März 1978 in eine mir völlig unbekannte Gerichtsbarkeit ein, wurde zunächst am Landessozialgericht geschlagene fünf Monate lang eingewiesen und trat dann meinen ersten Kammervorsitz beim Sozialgericht Oldenburg an, was mich ein wenig ärgerte, weil mein Zuhause ja in Aurich war. Später war ich dem damaligen Präsidenten dankbar dafür, dass er mich erst in Oldenburg einsetzte. 
Dann begann der Berufsweg fachlich mit einem Sprung ins kalte Wasser und privat mit der ersten von vielen Trennungen?

Ja, denn die ersten Schritte als Kammervorsitzende waren so leicht nicht - trotz der langen Einweisung beim Landessozialgericht. Die Trennung, die ich mit einer Tätigkeit in Aurich hatte vermeiden wollen, hatte aber auch ihre Vorteile in dieser beruflich angespannten Situation: Ich war die Woche über in Oldenburg und konnte mich dort voll auf meine Arbeit konzentrieren. Mein Mann war in Aurich Rechtsanwalt.

Nach 13 Monaten wurde ich endlich an das Sozialgericht Aurich versetzt und dort schließlich im März 1981 zur Richterin am Sozialgericht ernannt. Das Sozialrecht und Sozialversicherungsrecht war mir inzwischen längst ans Herz gewachsen. Ich war dankbar dafür, Richterin sein zu dürfen. Die mündlichen Verhandlungen waren stets ein Highlight des Berufsalltags. Ich hatte an dem kleinen Auricher Sozialgericht Gelegenheit, in relativ kurzer Zeit eine Reihe verschiedener Rechtsgebiete zu bearbeiten.
Die Erprobungszeit betrug ein ganzes Jahr, und es war schon eine schwierige Entscheidung für mich und meinen Mann, mich für ein Jahr abordnen zu lassen. Wir hatten in Aurich inzwischen ein Haus gekauft.

Die Woche würde ich - wie schon in der Einweisungszeit - in Celle sein müssen; mein Mann war inzwischen Notar geworden und sehr beschäftigt. Ich würde ihn wieder nur an den Wochenenden sehen. All diese Nachteile ließen wir jedoch nicht gelten. Ich wollte unbedingt zur Erprobung und mein Mann hat mich unterstützt. Er war und ist mein ganz großer Förderer.

Die Arbeit als Berichterstatterin in einem Senat gefiel mir gut, und so war es nicht schwer zu entscheiden, meinen Berufsweg mit 36 Jahren als Richterin am Landessozialgericht fortzusetzen.

Der Weg an die Spitze eines Landesobergerichts führt über viele Stationen in Rechtsprechung und Verwaltung. Wie ging es für Sie weiter?

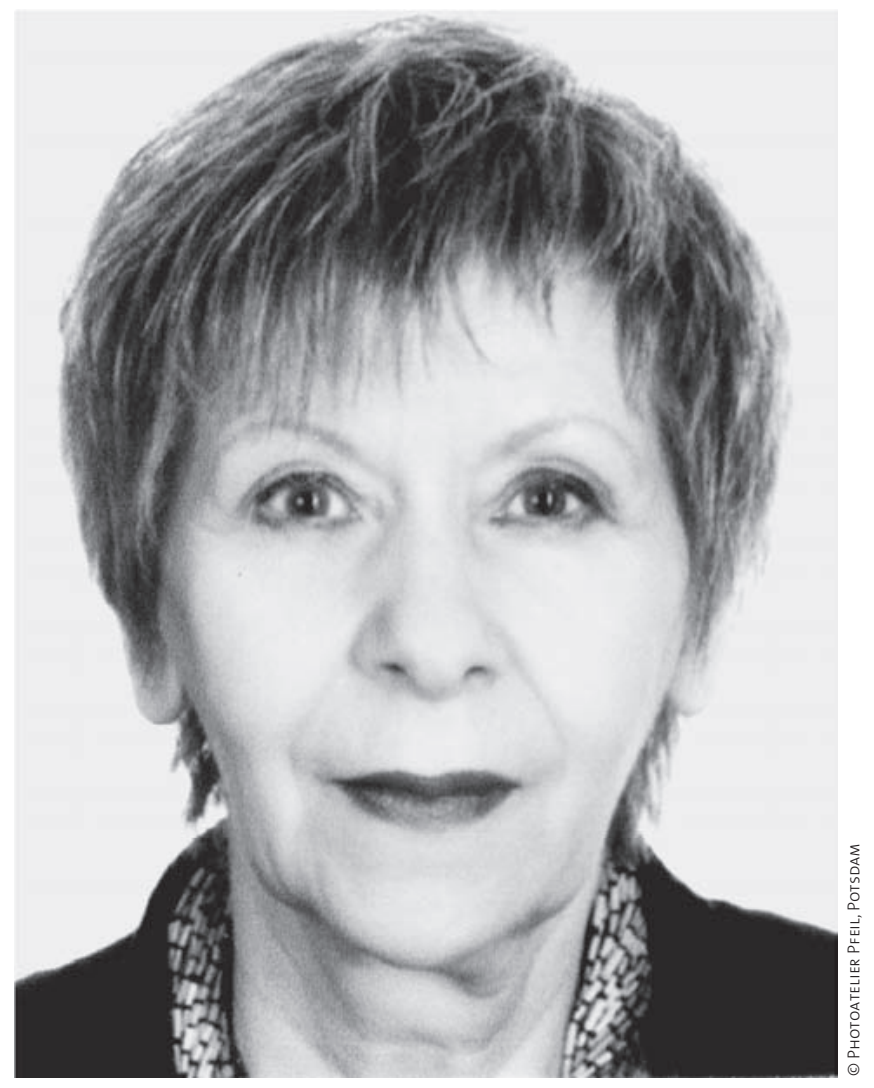

Das muss eine schöne Zeit für Sie gewesen sein: den Wunschberuf in der bevorzugten Fachrichtung in einem überschaubaren Gericht ausüben und als Ehepaar an einem Ort zusammenleben zu können. Was hat Sie bewegt, diese Situation zu verändern?

Irgendwann warb die Präsidialrichterin des Landessozialgerichts dafür, dass sich junge Richterinnen und Richter in der Berufungsinstanz erproben lassen sollten. Ich fühlte mich angesprochen und meldete mich.
Ein dritter Förderer war auf den Plan getreten, nämlich der Präsident des Landessozialgerichts, der mich nicht lange nach der Ernennung fragte, ob ich mir vorstellen könnte, in der Gerichtsverwaltung mitzuarbeiten. Und ob ich konnte! Ich war zunächst Präsidialrichterin für Justitiarangelegenheiten und schließlich Präsidialrichterin für Personalangelegenheiten. Wenn Sie fragen, warum der Präsident damals auf mich kam: Vielleicht hatte er gesehen, dass ich gut mit Menschen umgehen kann. 
Diese Präsidialrichterzeit war eine wunderbare Schule für mein späteres Berufsleben. Nach dem frühen Tod des Präsidenten musste ich gemeinsam mit dem Vizepräsidenten, der nicht sehr eingeweiht war in Verwaltungssachen, die Geschicke des Landessozialgerichts und der Sozialgerichtsbarkeit in Niedersachsen leiten. Mein Verwaltungshandwerkszeug habe ich damals gelernt.

Dann haben Sie die durch einen tragischen Anlass sich bietende Chance einer Bewährung in hoher Funktion offenbar gut genutzt. Wann wurde Ihnen die erste eigene Leitung eines Gerichts übertragen?

Im Januar 1989, als ich Direktorin des Sozialgerichts Oldenburg wurde und damit wieder in die Nähe von Aurich rückte. Dort konnte ich das Gelernte umsetzen. Die Zeit am Sozialgericht Oldenburg war besonders schön. Und doch zog es mich an das Sozialgericht Hannover, das größte niedersächsische Sozialgericht.

Auch während der Zeit in Hannover wohnte ich in einer kleinen möblierten Wohnung an meinem Dienstort und fuhr nur am Wochenende nach Aurich.

\section{Warum haben Sie die Mehrbelastung und das erneute Ge- trenntleben auf sich genommen?}

Die Verbindung von Richteramt und Verwaltungstätigkeit schätzte ich sehr. Beim Sozialgericht Hannover waren schwierige Verwaltungsaufgaben zu erfüllen. Es war eine Herausforderung, dort zu arbeiten. Großstadtgerichte sind anders als die kleinen Gerichte an der Peripherie.

Eigentlich hätte ich noch ein wenig mehr Zeit dort verbringen wollen. Im Dezember 1995 aber kam ein überraschender Anruf aus Bremen, wo der Präsident des Landessozialgerichts in Pension gegangen war. Sollte ich nun das Bundesland wechseln? Sollte ich Präsidentin werden? Ich dachte, was andere dir zutrauen, traust du dir selber auch $\mathrm{zu}$.

Ich stellte mich der Justizdeputation vor und wurde am 1. April 1996 zur Präsidentin des kleinsten Landessozialgerichts der Republik ernannt.

In Bremen, das merkte ich bald, war vieles anders, in der Justizverwaltung und auch sonst. Es waren interessante Erfahrungen. Es gab viel zu tun, einiges zu ändern. Ich hatte eine schöne Zeit in Bremen und habe mir den hanseatischliberalen Wind gern um die Nase wehen lassen.

Ich wohnte in der schönen Parkallee und merkte, wie viel kürzer im Verhältnis zu Hannover der Weg nach Aurich geworden war.

Präsidentin des LSG, in schöner Umgebung, wohnortnah manch eine(r) würde denken, nun angekommen zu sein. Stattdessen schulterten Sie eine neue, schwere Aufgabe, von der Sie wussten, dass Sie nicht nur auf Befürwortung und Unterstützung treffen würden?

Stimmt. So hätte ich denn in Bremen mein Berufsleben fortsetzen und beschließen können, wenn nicht Ende 1997 die
Idee eines gemeinsamen Landessozialgerichts Niedersachsen-Bremen geboren worden wäre.

Von dem Gedanken eines gemeinsamen Obergerichts für Bremen und Niedersachsen war ich sofort fasziniert. Viele Gerichtsangehörige beider Obergerichte eher nicht.

In Vorbereitung der Fusion sollte ich das Landessozialgericht Niedersachsen in Celle übernehmen, nachdem der dortige Präsident ausgeschieden war. So ist es auch gekommen. Am 1. April 1999 wurde ich zur Präsidentin des Landessozialgerichts Niedersachsen ernannt. Von dort aus begleitete ich das Unternehmen „Gemeinsames Landessozialgericht“, das das erste in der Bundesrepublik werden sollte. Es war ein anstrengender und schwieriger Weg bis zur Fusion am 1. April 2002. Es gab viele Widerstände. Es musste permanent Überzeugungsarbeit geleistet werden, bis der Staatsvertrag endlich unterschrieben war.

Am 1. April 2002 hatten Bremen und Niedersachsen ein gemeinsames Landessozialgericht mit Hauptsitz in Celle und Zweigstelle in Bremen.

Als Präsidentin mussten Sie sich jetzt neben der häuslichen Trennung auch beruflich örtlich „aufteilen“? Im Bereich der Rechtsprechung führte die Sozialgerichtsbarkeit seinerzeit noch ein eher unauffälliges, zurückgezogenes Leben. War die Hauptarbeit mit der Fusion getan?

Im Hinblick auf diese Fusionierung hatten mein Mann und ich unseren Hauptwohnsitz in Bremen genommen; das war nicht ohne Risiko, weil wir nicht sicher sein konnten, dass das gemeinsame Gericht auch wirklich gegründet würde.

Nun war auch mein Mann „Fahrschüler“; wir teilten uns die Last des Fahrens.

Die Zusammenführung der beiden Gerichtsbarkeiten war auch nach der Fusion der Landessozialgerichte herausfordernd. Haupt- und Zweigstelle mussten betreut, das Zusammenwachsen gefördert werden. Ich erinnere mich an schwierige Präsidiumssitzungen. Aber allmählich schien zusammenzuwachsen, was zuvor nicht zusammengehört hatte. Eine riesengroße Herausforderung, die nur im ehemaligen gemeinsamen Oberverwaltungsgericht Schleswig-HolsteinNiedersachsen ein Beispiel hatte.

Und im Januar 2005 kam eine weitere Herausforderung hinzu: Hartz IV! Arbeitslosenhilfe und Sozialhilfe waren zusammengelegt worden. Die Sozialgerichtsbarkeit hatte darum gekämpft, die Zuständigkeit zu erhalten, und hatte sie erhalten. Prognosen über die zukünftige Geschäftsbelastung waren vorsichtig und - wie sich herausgestellt hat - völlig unzutreffend. Die Geschäftsbelastung stieg in Niedersachsen sprunghaft. Bremen hatte sich dafür entschieden, die Hartz-IV-Verfahren bis Ende 2008 der Verwaltungsgerichtsbarkeit zuzuweisen. Die Sozialgerichtsbarkeit in Niedersachsen war innerhalb kürzester Zeit - wie die in anderen Bundesländern auch - überflutet und brauchte personelle Unterstützung, die sie zeitversetzt auch bekam. Die Vergrößerung der Gerichtsbarkeit war enorm, sie verjüngte sich enorm und änderte ihr Gesicht in dramatischer Weise. Und sie wurde in 
der Öffentlichkeit plötzlich bekannt. Alle Welt hatte Interesse an ihr und den Hartz-IV-Verfahren.

Das klingt in Ihrer Schilderung ambivalent: überrollt und gleichzeitig enthusiastisch?

Das hören Sie richtig heraus: Die ersten Jahre nach Einführung von Hartz IV waren schwierig und spannend zugleich. Solch eine Veränderung hatte eine Gerichtsbarkeit noch nicht erlebt. Und in dieser spannenden, aufregenden Zeit durfte ich Präsidentin eines um vier Senate vergrößerten Landessozialgerichts sein und einer immer noch wachsenden Sozialgerichtsbarkeit!

Andererseits zeichnete sich gerade durch diese immense Belastung der Gerichtsbarkeit durch Maßnahmen der Politik die Notwendigkeit für mich ab, politische Prozesse und Gesetzgebungsvorhaben fachlich und aus justizieller Perspektive zu begleiten. So gehörte ich am 25. Januar 2006 zu den Gründer(inne)n des Deutschen Sozialgerichtstags e.V. (DSGT), den ich seitdem als Präsidentin führen darf. Dieses Gremium unterbreitet nach dem Beispiel des seit Langem etablierten Verkehrsgerichtstags dem Gesetzgeber Vorschläge für künftige Reformen, auch zur Straffung des Verfahrensrechts. Ich habe damit eine ungemein reizvolle Aufgabe übernommen. Im November 2006 fand der erste Deutsche Sozialgerichtstag in Berlin-Köpenick statt. Seit 2008 lädt der DSGT alle zwei Jahre nach Potsdam zum Bundeskongress ein.

Wohin der Weg dann auch bald führte. Warum nach Potsdam? Die Fusion in Niedersachen war geschafft, in Potsdam stand eine bevor, die Klageflut nach „Hartz IV“ war immer noch anschwellend und beruflich so weit entfernt von zuhause wie zuvor nie?

Karrierewege und -ziele können und müssen zwar strukturiert und geplant beschritten und angesteuert werden. Wenn Offenheit und Neugier dazukommen, werden Sie feststellen, dass dies hilft, sich dabei nicht in Sackgassen zu manövrieren, sondern an Kreuzungen und Weggabelungen anzukommen. Oft entscheidet dann eine unerwartete Wendung - zur eigenen Überraschung - über den weiteren Weg. So ging es mir mit Potsdam.

Ich arbeitete viel in Niedersachsen und Bremen, hatte große Freude an meinen Aufgaben und dachte nicht im Mindesten daran, dass sich an meinem Standort noch etwas ändern würde. Das war ein Irrtum. Im Herbst 2008 begannen erste Gespräche über einen Wechsel an das Landessozialgericht Berlin-Brandenburg.

Ich hatte schon früher einmal mit Potsdam geliebäugelt, als das dort ansässige Landessozialgericht noch selbständig war. Für Preußen hatte ich mich immer interessiert und nun wurde ich gefragt, ob ich mir vorstellen könnte, nach Potsdam zu kommen. Das war natürlich ein sehr großer Schritt weg von Celle und vor allen Dingen von Bremen. Aber ich habe nicht lange gezögert, um mir auch das vorzustellen. Bei einem Aufenthalt an der Richterakademie in Wustrau - das war noch vor dem ersten Gespräch über das Landessozialge- richt Berlin-Brandenburg - hatte ich mich ein bisschen in Brandenburg verliebt. Und nun bin ich schon beinahe zwei Jahre in Potsdam Präsidentin des Landessozialgerichts und habe noch zu keiner Sekunde bereut, hierher gekommen zu sein.

Beruf und Privatleben sind zwar noch ein bisschen schwieriger miteinander zu verbinden als früher schon. Aber ich bin unendlich dankbar für die Bereicherung, die mir der berufliche Wechsel verschafft. Mein Mann und ich genießen es, an gemeinsamen Wochenenden in Potsdam diese wunderbare Stadt zu erkunden, Brandenburg kennenzulernen und uns in der Bundeshauptstadt umzutun. Dienstlich gibt es eine Menge Aufgaben. Ich lerne so viele interessante Menschen und ihre Geschichte kennen. Und ich habe, was ich mir in Niedersachsen nicht hätte vorstellen können, Zugang zum Juristinnenbund gefunden.

Ich vergesse es nicht, wie auf meiner offiziellen Amtseinführung die damals stellvertretende Vorsitzende im Landesverband Brandenburg, Notarin Dr. Ursula Bücker, so herzlich auf mich zukam, mich mit dem berühmten djb-Becher beschenkte und zwei Flaschen Brandenburger Wein. Ich besuchte das nachfolgende Sommerfest des djb, das Sie und die Vorstandsmitglieder so liebevoll arrangiert und interessant gestaltet hatten. Mir fiel das alters- und berufsmäßig breite Spektrum der Mitglieder auf. Die Gespräche, die ich auf dieser ersten Veranstaltung führen konnte, waren offen, unprätentiös, unverkrampft und niveauvoll. Ich fühlte mich dem Landesverband sofort verbunden und trat ihm bei. Ganz stolz bin ich darauf, inzwischen stellvertretende Vorsitzende zu sein.

Und wir darauf, dass Sie sich so spontan auch auf diese Aufgabe eingelassen haben. Mit der Präsidentin eines Obergerichtes ist der djb in Brandenburg sehr prominent repräsentiert. Die Vorbildwirkung für junge Kolleginnen ist enorm. Vom jungen Mädchen, das den Deutschunterricht schätzt, zu einer Gerichtspräsidentin - das ist trotz vieler Gymnasiastinnen mit vergleichbaren Interessen, vieler Studentinnen und Absolventinnen der Rechtswissenschaft sowie seit Jahren hoher und inzwischen überwiegender Einstellung junger Frauen für den richterlichen Dienst leider immer noch alles andere als selbstverständlich. Der Bundeskongress des djb vom 22. bis 25. September in Potsdam widmet sich diesem Thema unter dem Titel: „Gläserne Decke für Frauen: Der öffentliche Dienst als Vorreiter für Gleichberechtigung?" Was halten Sie für den entscheidenden Impuls auf Ihrem beruflichen Werdegang?

Wenn ich mein Berufsleben Revue passieren lasse, bin ich selbst erstaunt über die häufigen Änderungen und Standortwechsel. Von der Studentin bis zur Präsidentin eines der größten Landessozialgerichte ist es ein langer Weg gewesen. Ich habe die mir gebotenen Chancen immer genutzt. Ich habe mir eine Menge zugetraut und es wurde mir von anderen viel zugetraut. Viele meiner Entscheidungen waren mutig, vielleicht sogar wagemutig. 
Verwirklichen kann man einen solchen Weg nicht ohne Unterstützung und Förderung von vielen Seiten, Männern und Frauen. Das fängt in der eigenen Familie an. Wichtig und unverzichtbar aber scheint mir, dass sich Frauen etwas zutrauen, nicht immer nur die Nachteile und Risiken sehen, sondern auch bereit sind, gewisse Entbehrungen auf sich zu nehmen.

Sich einbringen durch Leistung, sich selbstbewusst einmischen und auf sich aufmerksam machen ist ein Erfolgsrezept. Dabei weiß ich sehr wohl, dass dies mit Kindern, die ich nicht habe, durchaus nicht leicht ist.

In der Justiz, denke ich, sind die Chancen für Frauen, in Führungspositionen zu gelangen, gut, zumal in der Sozialgerichtsbarkeit. Ich bin stolz darauf zu berichten, dass die Hälfte der Landessozialgerichte von einer Frau geleitet wird. Und das ist gut so.

Dann steht die Sozialgerichtsbarkeit deutlich besser da als die große Ordentliche Gerichtsbarkeit mit nur fünf Frauen an der Spitze unter 24 Oberlandesgerichten, zwei davon übrigens im Norden, wo der Himmel höher und die Decken nicht undurchlässig zu sein scheinen. Zur Nachahmung empfohlen?

Ja, jedenfalls, denn Frauen führen anders. Das muss nicht heißen, dass sie immer besser führen als Männer. Sie führen aber teamorientierter, sind kommunikativer, vielleicht auch manchmal fleißiger. Sie sind in der Regel jedenfalls weniger eitel und selbstbezogen, weniger an Statussymbolen interessiert, sie üben die ihnen anvertraute Macht menschlicher aus. Ausnahmen bestätigen aber auch hier die Regel.

Mein Weg nach Potsdam war lang. Trotz aller Überraschung und Ungeplantheit empfinde ich eine gewisse innere Konsequenz, dass ich jetzt hier bin und mit Ihnen zur Veröffentlichung in der djbZ über meinen Werdegang spreche.

\section{Liebe Frau Paulat, haben Sie herzlichen Dank für das offene Gespräch.}

\section{Impressum}

\section{Schriftleitung:}

Anke Gimbal, Rechtsassessorin (V.i.S.d.P.)

Juliane Lindner

Redaktionsanschrift:

Deutscher Juristinnenbund e. V.

Anklamer Str. 38

10115 Berlin

Telefon: 030 443270-0

Telefax: 030 443270-22

E-Mail: geschaeftsstelle@djb.de

\section{Druck und Verlag:}

Nomos Verlagsgesellschaft mbH \& Co. KG

Waldseestr. 3-5

D-76530 Baden-Baden

Telefon: 07221 2104-0

Telefax: 07221 2104-27

\author{
Anzeigenverwaltung und \\ Anzeigenannahme: \\ Sales friendly, Verlagsdienstleistungen \\ Bettina Roos \\ Siegburgerstr. 123 \\ D-53229 Bonn \\ Telefon: 0228 97898-o \\ Telefax: 0228 97898-20 \\ E-Mail: roos@sales-friendly.de
}

Die Zeitschrift sowie alle in ihr enthaltenen einzelnen Beiträge und Abbildungen sind urheberrechtlich geschützt. Jede Verwertung, die nicht ausdrücklich vom Urheberrechtsgesetz zugelassen ist, bedarf der vorherigen Zustimmung des Verlages.

Namentlich gekennzeichnete Artikel müssen nicht die Meinung des Herausgebers oder der Schriftleitung wiedergeben. Unverlangt eingesendete Manuskripte - für die keine Haftung übernommen wird - gelten als Veröffentli- chungsvorschlag zu den Bedingungen des Verlags. Es werden nur unveröffentlichte Originalarbeiten angenommen. Die Verfasser erklären sich mit einer nicht sinnentstellenden redaktionellen Bearbeitung einverstanden.

Erscheinungsweise:

vierteljährlich

Bezugspreis 2011:

jährlich 48,-€, Einzelheft 13,-€

Alle Preise zzgl. Vertriebs-Direktbeordnungsgebühren inkl. MWSt.;

Bestellungen nehmen entgegen:

Der Buchhandel und der Verlag; Kündigung jeweils drei Monate zum Kalenderjahresende. Zahlungen jeweils im Voraus an: Nomos Verlagsgesellschaft, Postbank Karlsruhe, Konto 7363651 (BLZ 66010075) oder Stadtsparkasse Baden-Baden, Konto 5002266 (BLZ 66250030). 